\title{
Global finite-time stability characterized through a local notion of homogeneity
}

\author{
Arturo Zavala-Río, Isabelle Fantoni
}

\begin{abstract}
In this work, a notion of local homogeneity is formally defined. Important results involving homogeneous functions or vector fields are reformulated in the consequent local context. In particular, global finite-time stability is characterized through the proposed notion of local homogeneity. As an application of the developed analytical setting, a global finite-time stabilization scheme for robot manipulators with bounded inputs is presented. The developed framework and results prove to be useful to relax analytical and synthesis constraints imposed or generated by homogeneity requirements.
\end{abstract}

Index Terms-Finite-time stability, local homogeneity, bounded inputs.

\section{INTRODUCTION}

Global finite-time stability of the origin, as an equilibrium of a dynamical system, has been characterized through homogeneity for instance in [1, Theorem 7.1] (see Appendix A; sufficient versions of this result have been presented in [2, Lemma 1] and [3, Corollary 5.4]). Requiring homogeneity of the vector field involved in the state-space representation of a system dynamics may however result restrictive. This is the case, for instance, when the design of finitetime stabilizers is aimed in a constrained input context where the control signal is required to take values within a bounded range. Through a bounded feedback algorithm, the resulting closed-loop vector field cannot be homogeneous in the coordinate framework naturally defined by the state variables [1]. This brings to the fore the convenience to state a new notion of local homogeneity through which such an analytical restriction can be relaxed. Such a convenience has also been apparent in [4], where stabilization of a PVTOL aircraft is achieved through (unbounded) control expressions that give rise to a partially homogeneous closed loop structure. Through such a design procedure the authors achieved considerably faster closed-loop responses compared to those arisen with control expressions rendering (completely) homogeneous the closed-loop system. Nevertheless, a local notion of homogeneity through which the mentioned special cases acquire a formal sense has not yet been proposed. Indeed, the traditional coordinate-dependent definition of homogeneous functions and vector fields, stated for instance in [1], [3], [5], [6], requires to hold on $\mathbb{R}^{n}$ and for the whole dilation. Moreover, the existing analytical results on homogeneous functions or vector fields consider that such a global feature of homogeneity is fulfilled. Thus, such results do not necessarily hold and cannot even be applied if the homogeneity property is permitted to be satisfied only locally in some sense.

Such limitations motivated the present study which aims at: stating a new concept of local homogeneity through a formal definition, developing an analytical framework where some previous results that involve homogeneous functions or vector fields are reformulated in

A. Zavala-Río (corresponding author) is with the Instituto Potosino de Investigación Científica y Tecnológica, Camino a la Presa San José 2055 Lomas 4a. Sección 78216, San Luis Potosí, S.L.P., Mexico. Tel.: +52 - 444 - 834.2000. Fax: +52 - 444 - 834.2010. E-mail: azavala@ipicyt.edu.mx .

I. Fantoni is with the Université de Technologie de Compiègne - CNRS, UMR 7253 Heudiasyc, BP 20529, 60200 Compiègne, France. Tel.: +33-(0)3.44.23.49.36. Fax: +33-(0)3.44.23.44.77. E-mail: isabelle.fantoni@hds.utc.fr . the defined local context, and stating a formal characterization of global finite-time stability through the proposed notion of local homogeneity. As an application of the developed analytical framework, a global finite-time stabilization scheme for robot manipulators with bounded inputs is presented.

\section{A LOCAL NOTION OF HOMOGENEITY}

We begin by formally defining a local notion of homogeneity. Analogously to the case of homogeneous functions and vector fields [1], [3], [5], [6], the local concept is stated in terms of family of dilations $\delta_{\varepsilon}^{r}$, defined as $\delta_{\varepsilon}^{r}(x)=\left(\varepsilon^{r_{1}} x_{1}, \ldots, \varepsilon^{r_{n}} x_{n}\right), \forall x \in \mathbb{R}^{n}$, $\forall \varepsilon>0$, where $r=\left(r_{1}, \ldots, r_{n}\right)$, with the dilation coefficients $r_{1}, \ldots, r_{n}$ being positive real numbers. Subsequently, we denote $\mathbb{R}_{>0}^{n}$, resp. $\mathbb{R}_{\geq 0}^{n}$, the set of vectors $x \in \mathbb{R}^{n}$ such that $x_{i}>0$, resp. $x_{i} \geq 0, \forall i=1, \ldots, n$, and $0_{n}$ the origin of $\mathbb{R}^{n}$. Given $r \in \mathbb{R}_{>0}^{n}$, a neighborhood of the origin $D \subset \mathbb{R}^{n}$ will be said to be $\delta_{\varepsilon}^{r}$-connected if, for every $x \in D, \delta_{\varepsilon}^{r}(x) \in D$ for all $\varepsilon \in(0,1)$.

Definition 2.1: Given $r \in \mathbb{R}_{>0}^{n}$, a function $V: \mathbb{R}^{n} \rightarrow \mathbb{R}$, resp. vector field $f: \mathbb{R}^{n} \rightarrow \mathbb{R}^{n}$, is locally homogeneous of degree $\alpha$ with respect to the family of dilations $\delta_{\varepsilon}^{r}$-or equivalently, it is said to be locally $r$-homogeneous of degree $\alpha$ - if there exists a $\delta_{\varepsilon}^{r}$-connected open neighborhood of the origin $D \subset \mathbb{R}^{n}$-referred to as the domain of homogeneity — such that

$$
V\left(\delta_{\varepsilon}^{r}(x)\right)=\varepsilon^{\alpha} V(x)
$$

resp.

$$
f\left(\delta_{\varepsilon}^{r}(x)\right)=\varepsilon^{\alpha} \delta_{\varepsilon}^{r}(f(x))
$$

for every $x \in D$ and all $\varepsilon>0$ such that $\delta_{\varepsilon}^{r}(x) \in D$.

Observe that a locally $r$-homogeneous function or vector field with domain of homogeneity $D \subset \mathbb{R}^{n}$ is not necessarily $r$-homogeneous, unless $D=\mathbb{R}^{n}$, while an $r$-homogeneous function or vector field is locally $r$-homogeneous for any $\delta_{\varepsilon}^{r}$-connected open neighborhood of the origin $D \subset \mathbb{R}^{n}$. Let us further note that a locally $r$-homogeneous function $V: \mathbb{R}^{n} \rightarrow \mathbb{R}$, or vector field $f: \mathbb{R}^{n} \rightarrow \mathbb{R}^{n}$, satisfies Eq. (1), or Eq. (2) respectively, for every $x \in D \backslash\left\{0_{n}\right\}$ and all $\varepsilon \in\left(0, \bar{\varepsilon}_{x}^{D}\right)$, where $\bar{\varepsilon}_{x}^{D}=\sup \left\{\varepsilon \in \mathbb{R}_{>0}: \delta_{\varepsilon}^{r}(x) \in D\right\}$. Actually, for every $x \in D \backslash\left\{0_{n}\right\}$, the subset $\mathcal{R}_{x}^{D}=\left\{\delta_{\varepsilon}^{r}(x) \in \mathbb{R}^{n}: \varepsilon \in\left(0, \bar{\varepsilon}_{x}^{D}\right)\right\} \subset D$ is the ray segment through $x$ contained in $D$-which comprehends, and is consequently equivalent to, the (whole) ray through $x[6, \S 3.2]$ when $\bar{\varepsilon}_{x}^{D}=\infty$ - Let us further consider the set

$$
S_{c}^{n-1}=\left\{x \in \mathbb{R}^{n}:\|x\|=c\right\}
$$

for some $c>0$ such that $S_{c}^{n-1} \subset D$ (such a positive value $c$ exists in view of the open nature of $D$ ). The reformulation of some fundamental analytical results, originally stated under the consideration of $r$-homogeneous functions and vector fields (in the traditional sense), leans on some important properties that are kept on the domain of homogeneity in the local context. One of such properties is the following.

Remark 2.1: A locally $r$-homogeneous function $V$, with domain of homogeneity $D$, is entirely defined by its values on $S_{c}^{n-1}$. This is a consequence of the next fundamental analytical fact. 
Remark 2.2: The map $\phi:(y, \varepsilon) \mapsto \delta_{\varepsilon}^{r}(y)$ is an infinitely continuously differentiable bijection from $\left\{(y, \varepsilon): y \in S_{c}^{n-1}, \varepsilon \in\left(0, \bar{\varepsilon}_{y}^{D}\right)\right\}$ onto $D \backslash\left\{0_{n}\right\}$. Moreover, its inverse, $(y, \varepsilon)=\phi^{-1}(x)$, is such that $\varepsilon \rightarrow 0$ as $x \rightarrow 0_{n}$ and $\varepsilon \rightarrow \bar{\varepsilon}_{y}^{D}$ as $x \rightarrow \partial D$.

Indeed, observe that for every $y \in S_{c}^{n-1}$, by taking $\varepsilon=e^{t}, t \in \mathbb{R}$, and defining $A=\operatorname{diag}\left[r_{1}, \ldots, r_{n}\right]$, the ray segment $\mathcal{R}_{y}^{D}$ turns out to be the solution of the first order vector differential equation $\frac{d x}{d t}=A x$, with $x(0)=y$, for all $t \in\left(-\infty, \ln \bar{\varepsilon}_{y}^{D}\right)$. Thus, the ray segments $\mathcal{R}_{y}^{D}$ on $S_{c}^{n-1}$ are unique, i.e. $\mathcal{R}_{y_{1}}^{D} \cap \mathcal{R}_{y_{2}}^{D}=\emptyset, \forall y_{1}, y_{2} \in S_{c}^{n-1}$ such that $y_{1} \neq y_{2}$ (and actually $\bigcup_{y \in S_{c}^{n-1}} \mathcal{R}_{y}^{D}=D \backslash\left\{0_{n}\right\}$ ). Then, for every $x \in D \backslash\left\{0_{n}\right\}$, there is one and only one $y \in S_{c}^{n-1}$ such that $x \in \mathcal{R}_{y}^{D}$. Moreover, since $\varepsilon: t \mapsto e^{t}$ states a one-to-one relation from $\left(-\infty, \ln \bar{\varepsilon}_{y}^{D}\right)$ onto $\left(0, \bar{\varepsilon}_{y}^{D}\right)$, for every $x \in \mathcal{R}_{y}^{D}$ there is one and only one $\varepsilon \in\left(0, \bar{\varepsilon}_{y}^{D}\right)$ such that $x=\delta_{\varepsilon}^{r}(y)$. Thus, $\phi:(y, \varepsilon) \mapsto \delta_{\varepsilon}^{r}(y)$ is concluded to bijectively map $\left\{(y, \varepsilon): y \in S_{c}^{n-1}, \varepsilon \in\left(0, \bar{\varepsilon}_{y}^{D}\right)\right\}$ onto $D \backslash\left\{0_{n}\right\}$ and (from the differentiability properties of the ray segments as solutions of the considered linear vector differential equation) to be infinitely continuously differentiable. Observe that since $\delta_{e^{t}}^{r}(y) \rightarrow$ $0_{n} \Longrightarrow t \rightarrow-\infty \Longleftrightarrow e^{t} \rightarrow 0$, and $\delta_{e^{t}}^{r}(y) \rightarrow \partial D \Longrightarrow$ $t \rightarrow \ln \bar{\varepsilon}_{y}^{D} \Longleftrightarrow e^{t} \rightarrow \bar{\varepsilon}_{y}^{D}$, we conclude that the inverse of $\phi$, $(y, \varepsilon)=\phi^{-1}(x)$, is such that $\varepsilon \rightarrow 0$ as $x \rightarrow 0_{n}$ and $\varepsilon \rightarrow \bar{\varepsilon}_{y}^{D}$ as $x \rightarrow \partial D$.

Another fundamental property being essential in the analytical setting developed in this section is the following one.

Remark 2.3: The directional derivative of a smooth function $V$ being locally $r$-homogeneous of degree $\alpha$ with domain of homogeneity $D_{V} \subset \mathbb{R}^{n}$, in the direction of a vector field $f$ being locally $r$ homogeneous of degree $k$ with domain of homogeneity $D_{f} \subset \mathbb{R}^{n}$, is locally $r$-homogeneous of degree $k+\alpha$ with domain of homogeneity $D=D_{V} \cap D_{f}$. Also, if $\frac{\partial V}{\partial x} f(x)<0$ on $S_{c}^{n-1}$, then $\frac{\partial V}{\partial x} f(x)<0$, $\forall x \in D \backslash\left\{0_{n}\right\}$.

This is corroborated following a procedure analogue to the one developed in [7] in the homogeneous case, applied to every $x \in D$ and for all $\varepsilon>0$ such that $\delta_{\varepsilon}^{r}(x) \in D$.

The next result is a reformulation in the local homogeneity context of Rosier's theorem [3, Theorem 5.8] (see Appendix A) originally stated in [7, Theorem 2] under the consideration of $r$-homogeneous continuous vector fields. Subsequently, we denote $\mathcal{C}^{m}(\mathcal{A}, \mathcal{E})$ the set of $m$-times continuously differentiable functions from $\mathcal{A}$ to $\mathcal{E}$ (subsets with nonempty interior of some vector spaces $\mathbb{A}$ and $\mathbb{E}$ respectively).

Theorem 2.1: Let $f$ be a continuous vector field on $\mathbb{R}^{n}$ such that the origin is a globally asymptotically stable equilibrium of the $n$-th order autonomous system $\dot{x}=f(x)$. Assume that $f$ is locally $r$ homogeneous of degree $\alpha$ with domain of homogeneity $D \subset \mathbb{R}^{n}$. Then, for any positive integer $p$ and any $m>p \cdot \max \left\{r_{1}, \ldots, r_{n}\right\}$, there exists a (globally) positive definite function $V \in \mathcal{C}^{p}\left(\mathbb{R}^{n}, \mathbb{R}\right)$ being $r$-homogeneous of degree $m$, whose derivative along the system trajectories, $\dot{V}(x)=\frac{\partial V}{\partial x} f(x)$, is negative definite on $D$ and locally $r$-homogeneous of degree $m+\alpha$ with domain of homogeneity $D$.

Proof: Let $W: \mathbb{R}^{n} \rightarrow \mathbb{R}$ be the radially unbounded strict Lyapunov function proving the global asymptotic stability of the origin whose existence is guaranteed by Kurzweil's theorem, versions of which are given in [7, Theorem 1] (see Appendix A) and [8, Theorem 4.17]. Let $B_{b_{1}}=\left\{x \in \mathbb{R}^{n}:\|x\|<b_{1}\right\}$, $\bar{B}_{b_{2}}=\left\{x \in \mathbb{R}^{n}:\|x\| \leq b_{2}\right\}$, and $\bar{B}_{b_{1}}^{b_{2}}=\bar{B}_{b_{2}} \backslash B_{b_{1}}=\{x \in$ $\left.\mathbb{R}^{n}: b_{1} \leq\|x\| \leq b_{2}\right\}$, with $b_{2}>b_{1}>0$ such that $\bar{B}_{b_{1}}^{b_{2}} \subset D$ (such positive values exist in view of the open nature of $D$ ), and let $\Omega_{c_{1}}=\left\{x \in \mathbb{R}^{n}: W(x)<c_{1}\right\}, \bar{\Omega}_{c_{2}}=\left\{x \in \mathbb{R}^{n}: W(x) \leq c_{2}\right\}$, and $\bar{\Omega}_{c_{1}}^{c_{2}}=\bar{\Omega}_{c_{2}} \backslash \Omega_{c_{1}}=\left\{x \in \mathbb{R}^{n}: c_{1} \leq W(x) \leq c_{2}\right\}$, with $c_{2}>c_{1}>0$ such that $\bar{\Omega}_{c_{1}}^{c_{2}} \subset B_{b_{1}}$ (such positive values exist in view of the positive definite and continuous characters of $W$ ). Under the consideration of a function $a \in \mathcal{C}^{\infty}\left(\mathbb{R}, \mathbb{R}_{\geq 0}\right)$ satisfying $a(s)=0$ $\forall s \in\left(-\infty, c_{1}\right], a(s)=1 \forall s \in\left[c_{2}, \infty\right)$, and $a^{\prime}(s) \geq 0 \forall s \in \mathbb{R}$, let us define

$$
V(x) \triangleq\left\{\begin{array}{lc}
\int_{0}^{\infty} \frac{1}{t^{m+1}}(a \circ W)\left(t^{r_{1}} x_{1}, \ldots, t^{r_{n}} x_{n}\right) d t \\
\text { if } x \in \mathbb{R}^{n} \backslash\left\{0_{n}\right\} \\
0 & \text { if } x=0_{n}
\end{array}\right.
$$

Since $W$ is positive definite and radially unbounded, $V$ is well defined on $\mathbb{R}^{n}$. Moreover, notice on the one hand that, since $\bar{\Omega}_{c_{1}}^{c_{2}} \subset$ $\bar{\Omega}_{c_{2}} \subset B_{b_{1}}$, we have, for every $x \in \bar{B}_{b_{1}}^{b_{2}}$, that $\delta_{t}^{r}(x) \in \mathbb{R}^{n} \backslash B_{b_{1}} \subset$ $\mathbb{R}^{n} \backslash \bar{\Omega}_{c_{2}}, \forall t \geq 1$, and consequently $W\left(\delta_{t}^{r}(x)\right)>c_{2}, \forall t \geq 1$; and on the other hand that we can always find a (sufficiently small) value $l \in(0,1)$ such that, for every $x \in \bar{B}_{b_{1}}^{b_{2}}, \delta_{t}^{r}(x) \in \Omega_{c_{1}}, \forall t \in(0, l]$, and consequently $W\left(\delta_{t}^{r}(x)\right)<c_{1}, \forall t \in(0, l]$. Then, for every $x \in \bar{B}_{b_{1}}^{b_{2}}$,

$$
V(x)=\int_{l}^{1} \frac{1}{t^{m+1}}(a \circ W)\left(t^{r_{1}} x_{1}, \ldots, t^{r_{n}} x_{n}\right) d t+\frac{1}{m}
$$

Clearly, $V$ is positive on $\bar{B}_{b_{1}}^{b_{2}}$ and as many times continuously differentiable on $\bar{B}_{b_{1}}^{b_{2}}$ as $W$ is i.e., according to [7, Theorem 1] (see Appendix A), it is infinitely continuously differentiable on $\bar{B}_{b_{1}}^{b_{2}}$. Moreover, by considering $V\left(\delta_{\varepsilon}^{r}(x)\right)$, we get, after an obvious change of integration variable, that $V\left(\delta_{\varepsilon}^{r}(x)\right)=\varepsilon^{m} V(x), \forall x \in \mathbb{R}^{n}$, $\forall \varepsilon>0$, proving that $V$ in (4) is $r$-homogeneous of degree $m$ and, in accordance to Remark 2.1, that $V$ is positive on $\mathbb{R}^{n} \backslash\left\{0_{n}\right\}$-whence (global) positive definiteness of $V$ is concluded - and, moreover, infinitely continuously differentiable on $\mathbb{R}^{n} \backslash\left\{0_{n}\right\}$. According to Remark 2.3, we further conclude that $\dot{V}(x)=\frac{\partial V}{\partial x} f(x)$ is locally $r$-homogeneous of degree $m+\alpha$ with domain of homogeneity $D$. Furthermore, on $\bar{B}_{b_{1}}^{b_{2}}$, we have

$$
\begin{aligned}
& \frac{\partial V}{\partial x_{i}}(x)=\int_{l}^{1}\left[\frac{t^{r_{i}}}{t^{m+1}} a^{\prime}\left(W\left(t^{r_{1}} x_{1}, \ldots, t^{r_{n}} x_{n}\right)\right)\right. \\
&\left.\cdot \frac{\partial W}{\partial x_{i}}\left(t^{r_{1}} x_{1}, \ldots, t^{r_{n}} x_{n}\right)\right] d t
\end{aligned}
$$

Hence, from the satisfaction of Eq. (2) and the fact that $\bar{B}_{b_{1}}^{b_{2}} \subset \bar{B}_{b_{2}} \subset$ $D$-which implies that, for every $x \in \bar{B}_{b_{1}}^{b_{2}}, \delta_{t}^{r}(x) \in \bar{B}_{b_{2}} \backslash\left\{0_{n}\right\} \subset$ $D \backslash\left\{0_{n}\right\}, \forall t \in(0,1]$, and consequently that (2) is satisfied for all $t \in(0,1]$ - we get

$$
\begin{aligned}
\sum_{i=1}^{n} f_{i} \frac{\partial V}{\partial x_{i}}(x)=\int_{l}^{1} & {\left[\frac{1}{t^{k+m+1}} a^{\prime}\left(W\left(t^{r_{1}} x_{1}, \ldots, t^{r_{n}} x_{n}\right)\right)\right.} \\
& \left.\cdot \sum_{i=1}^{n}\left(f_{i} \frac{\partial W}{\partial x_{i}}\right)\left(t^{r_{1}} x_{1}, \ldots, t^{r_{n}} x_{n}\right)\right] d t
\end{aligned}
$$

and since $a^{\prime}(s)>0$ for some $s \in\left(c_{1}, c_{2}\right), \frac{\partial V}{\partial x} f(x)<0$ holds for all $x$ on $\bar{B}_{b_{1}}^{b_{2}}$. Hence, according to Remark 2.3 , we have that $\dot{V}(x)=\frac{\partial V}{\partial x} f(x)<0, \forall x \in D \backslash\left\{0_{n}\right\}$, which shows the negative definiteness of $\dot{V}$ on $D$. Finally, the fact that the infinitely continuousdifferentiability property of $V$ holds at the origin as well follows along the lines of the corresponding (last) part of the proof of Proposition 2 in [7].

The following result is a reformulation in the local homogeneity context - under the consideration of the coordinate-dependent family of dilations $\delta_{\varepsilon}^{r}$ - of Lemma 4.2 in [1] (see Appendix A; [1, Lemma 4.2] was stated for homogeneous continuous functions in a coordinate-free context; a coordinate-dependent particular version of [1, Lemma 4.2] was stated in [5, Lemma 2.2]).

Lemma 2.1: Suppose that, for every $i \in\{1,2\}, V_{i}$ is a scalar function being continuous on an open neighborhood $D_{i} \subset \mathbb{R}^{n}$ of the origin, and locally $r$-homogeneous of degree $\alpha_{i}>0$, with domain of homogeneity $D_{i}$. Suppose further that $V_{1}$ is positive definite on 
$D_{1}$. Let $D=D_{1} \cap D_{2}$ and $S_{c}^{n-1}$ as defined in (3) (for some $c>0$ such that $\left.S_{c}^{n-1} \subset D\right)$. Then, for every $x \in D$,

$$
c_{1}\left[V_{1}(x)\right]^{\alpha_{2} / \alpha_{1}} \leq V_{2}(x) \leq c_{2}\left[V_{1}(x)\right]^{\alpha_{2} / \alpha_{1}}
$$

with $c_{1}=\left[\min _{z \in S_{c}^{n-1}} V_{2}(z)\right] \cdot\left[\max _{z \in S_{c}^{n-1}} V_{1}(z)\right]^{-\alpha_{2} / \alpha_{1}}$ and $c_{2}=\left[\max _{z \in S_{c}^{n-1}} V_{2}(z)\right] \cdot\left[\min _{z \in S_{c}^{n-1}} V_{1}(z)\right]^{-\alpha_{2} / \alpha_{1}}$.

Proof: Since $\alpha_{1}, \alpha_{2}>0, V_{1}\left(0_{n}\right)=V_{2}\left(0_{n}\right)=0$ and (5) holds for $x=0_{n}$. Now, for any $x \in D \backslash\left\{0_{n}\right\}$, let $\varepsilon>0$ be such that $\delta_{\varepsilon}^{r}(x) \in S_{c}^{n-1}$. Such a value of $\varepsilon$ exists and is such that $0<\varepsilon<\bar{\varepsilon}_{x}^{D}$ since $\phi:(y, \varepsilon) \mapsto \delta_{\varepsilon}^{r}(y)$ bijectively maps $\left\{(y, \varepsilon): y \in S_{c}^{n-1}, \varepsilon \in\right.$ $\left.\left(0, \bar{\varepsilon}_{y}^{D}\right)\right\}$ onto $D \backslash\left\{0_{n}\right\}$ (recall Remark 2.2). Then, for every $i=1,2$, we have on the one hand, by local $r$-homogeneity of $V_{i}$ with domain of homogeneity $D_{i} \supset D$, that $V_{i}\left(\delta_{\varepsilon}^{r}(x)\right)=\varepsilon^{\alpha_{i}} V_{i}(x)$, and on the other hand that

$$
\min _{z \in S_{c}^{n-1}} V_{i}(z) \leq V_{i}\left(\delta_{\varepsilon}^{r}(x)\right) \leq \max _{z \in S_{c}^{n-1}} V_{i}(z)
$$

where the left- and right-most terms are well defined real values in view of the compact character of $S_{c}^{n-1}$. From these expressions we get

$$
\left[\frac{\min _{z \in S_{c}^{n-1}} V_{1}(z)}{V_{1}(x)}\right]^{1 / \alpha_{1}} \leq \varepsilon \leq\left[\frac{\max _{z \in S_{c}^{n-1}} V_{1}(z)}{V_{1}(x)}\right]^{1 / \alpha_{1}}
$$

and

$$
\min _{z \in S_{c}^{n-1}} V_{2}(z) \varepsilon^{-\alpha_{2}} \leq V_{2}(x) \leq \max _{z \in S_{c}^{n-1}} V_{2}(z) \varepsilon^{-\alpha_{2}}
$$

whence inequality (5) is obtained.

\section{CHARACTERIZING GLOBAL FINITE-TIME STABILITY} THROUGH LOCAL HOMOGENEITY

Consider an $n$-th order autonomous system

$$
\dot{x}=f(x)
$$

where $f: \mathbb{R}^{n} \rightarrow \mathbb{R}^{n}$ is continuous and $f\left(0_{n}\right)=0_{n}$, and let $x\left(t ; x_{0}\right)$ represent the system solution with initial condition $x\left(0 ; x_{0}\right)=x_{0}$. We begin by recalling the definition of a finite-time stable equilibrium [1], [2], [3], [9].

Definition 3.1: The origin is said to be a finite-time stable equilibrium of system (6) if it is Lyapunov stable and there exist an open neighborhood $\mathcal{N} \subset \mathcal{D}$ of the origin being positively invariant with respect to (6), and a positive definite function $T: \mathcal{N} \rightarrow \mathbb{R}_{\geq 0}$-called the settling-time function - such that $x\left(t ; x_{0}\right) \neq 0_{n}, \forall t \in\left[0, T\left(x_{0}\right)\right)$, $\forall x_{0} \in \mathcal{N} \backslash\left\{0_{n}\right\}$, and $x\left(t ; x_{0}\right)=0_{n}, \forall t \geq T\left(x_{0}\right), \forall x_{0} \in \mathcal{N}$. The origin is said to be a globally finite-time stable equilibrium of system (6) if it is finite-time stable with $\mathcal{N}=\mathcal{D}=\mathbb{R}^{n}$.

The following fundamental characterization, whose sufficient character was asserted in [2, Remark 1], follows directly from Definition 3.1 under the consideration of global asymptotic stability (in view of [1, Lemma 2.2]; see Appendix A).

Lemma 3.1: The origin is a globally finite-time stable equilibrium of system (6) if and only if it is globally asymptotically stable and finite-time stable.

The main result of the present section is now stated. ${ }^{1}$

Theorem 3.1: Suppose that $f$ in (6) is a locally $r$-homogeneous vector field of degree $k$ with domain of homogeneity $D \subset \mathbb{R}^{n}$. Then, the origin is a globally finite-time stable equilibrium of system (6) if and only if it is globally asymptotically stable and $k<0$.

Proof: Suppose that the origin is a globally asymptotically stable equilibrium of system (6) and $k<0$. By Theorem 2.1, there exists

\footnotetext{
${ }^{1}$ A partial version of Theorem 3.1 is found in [4] where no formal proof was furnished and no definition of a local notion of homogeneity was stated.
}

a continuously differentiable positive definite function $V: \mathbb{R}^{n} \rightarrow$ $\mathbb{R}$ being $r$-homogeneous of degree $m>\max \left\{r_{1}, \ldots, r_{n}\right\}$, whose derivative along the system trajectories, $\dot{V}=\frac{\partial V}{\partial x} f$, is continuous and negative definite on $D$ and locally $r$-homogeneous of degree $k+m$ with domain of homogeneity $D$. Applying Lemma 2.1 with $V_{1}=V, V_{2}=\dot{V}, D_{1}=\mathbb{R}^{n}$, and $D_{2}=D$, we have that

$$
-c_{1}[V(x)]^{\alpha} \leq \dot{V}(x) \leq-c_{2}[V(x)]^{\alpha}
$$

$\forall x \in D$, where $\alpha=(k+m) / m, c_{1}=-\left[\min _{z \in S_{c}^{n-1}} \dot{V}(z)\right]$. $\left[\max _{z \in S_{c}^{n-1}} V(z)\right]^{-\alpha}$, and $c_{2}=-\left[\max _{z \in S_{c}^{n-1}} \dot{V}(z)\right]$. $\left[\min _{z \in S_{c}^{n-1}} V(z)\right]^{-\alpha}$, with $S_{c}^{n-1}$ as defined in (3) (for some $c>0$ such that $\left.S_{c}^{n-1} \subset D\right)$. Observe that the negative definiteness of $\dot{V}$ implies on the one hand that $c_{1}>c_{2}>0$, and on the other that $\dot{V}$ is not constant whence we conclude that $k+m>0$-see for instance [1, Theorem 4.1] (see Appendix A), which applies in the local homogeneity context too-, and consequently $-m<k<0$. From this inequality, it follows that $0<k+m<m$ and therefore $0<\alpha<1$. Hence, by [9, Theorem 4.2] (see Appendix A) -in view of the satisfaction of the right-hand side inequality in (7) with $\alpha \in(0,1)$ - the origin turns out to be finite-time stable, and since its stability is globally asymptotical, we conclude from Lemma 3.1 that the finite-time stability of the origin is global, which proves the sufficiency of the statement.

Let us now suppose that the origin is globally finite-time stable. This implies that $0_{n}$ is Lyapunov stable and globally attractive, whence we conclude that the origin is globally asymptotically stable. Then, the existence of the previously described Lyapunov function $V$ satisfying (7) on $D$ is guaranteed (by Theorem 2.1 and Lemma 2.1). On the other hand, by [1, Lemma 2.2] (see Appendix A), there is a finite time $\tau \geq 0$ such that, for any $x_{0} \in \mathbb{R}^{n} \backslash\left\{0_{n}\right\}, x\left(t ; x_{0}\right) \in D$, $\forall t \geq \tau$, with $x_{\tau} \triangleq x\left(\tau ; x_{0}\right) \neq 0_{n}$. Thus, the application of the comparison principle [10, Chap. IX, §2] [11, Chap. I, §4] to inequality (7) yields

$$
\mu_{1}\left(t ; V\left(x_{\tau}\right)\right) \leq V\left(x\left(t ; x_{\tau}\right)\right) \leq \mu_{2}\left(t ; V\left(x_{\tau}\right)\right)
$$

$\forall t \geq \tau$, where, for every $i=1,2, \mu_{i}\left(t ; V\left(x_{\tau}\right)\right)$ is the solution of the first-order scalar differential equation $\dot{\mu}_{i}=-c_{i} \mu_{i}^{\alpha}$, with initial condition $\mu_{i}\left(\tau ; V\left(x_{\tau}\right)\right)=V\left(x_{\tau}\right)>0$. Obtained by integration, the exact expression of $\mu_{i}\left(t ; V\left(x_{\tau}\right)\right)$ is given by

$$
\mu_{i}\left(t ; V\left(x_{\tau}\right)\right)=\left\{\begin{array}{cc}
{\left[\left(V\left(x_{\tau}\right)\right)^{1-\alpha}+c_{i}(\alpha-1)(t-\tau)\right]^{-\frac{1}{\alpha-1}}} \\
\forall t \geq \tau, \alpha>1 \\
V\left(x_{\tau}\right) e^{-c_{i}(t-\tau)} \quad \forall t \geq \tau, \alpha=1 \\
{\left[\left(V\left(x_{\tau}\right)\right)^{1-\alpha}-c_{i}(1-\alpha)(t-\tau)\right]^{\frac{1}{1-\alpha}}} \\
\forall t \in\left[\tau, \tau+T_{i}\right) \\
0 \quad \forall t \geq \tau+T_{i}
\end{array}\right\} \alpha \in(0,1)
$$

with (settling time) $T_{i}=\left[\left(V\left(x_{\tau}\right)\right)^{1-\alpha}\right] /\left[c_{i}(1-\alpha)\right], i=1,2$. From (8) and (9), one sees that if $\alpha \geq 1$ then $\mu_{1}\left(t ; V\left(x_{\tau}\right)\right)>0, \forall t \geq$ $\tau$, and consequently $x\left(t ; x_{0}\right) \neq 0_{n}, \forall t \geq 0$, that is, the origin is not a globally finite-time stable equilibrium of system (6), while the assumed finite-time convergence takes place with $\alpha<1$. Thus, global finite-time stability of the origin implies $\alpha=(k+m) / m<1 \Longleftrightarrow$ $k<0$, which proves the necessary character of the statement.

Finite-time stability still holds under particular vector field perturbations (additional terms that render impossible the direct application of Theorem 3.1). The following special case is considered. Let

$$
\dot{x}=f(x)+\hat{f}(x)
$$


where $f: \mathbb{R}^{n} \rightarrow \mathbb{R}^{n}$ and $\hat{f}: \mathbb{R}^{n} \rightarrow \mathbb{R}^{n}$ are continuous vector fields such that $f\left(0_{n}\right)=\hat{f}\left(0_{n}\right)=0_{n}$, and recall the definition of $S_{c}^{n-1}$ in (3). The next result states a reformulation in the local homogeneity context of [12, Lemma 3] (see Appendix A), both extensions to the finite-time-stability framework of [7, Theorem 3].

Lemma 3.2: Suppose that $f$ in (10) is a locally $r$-homogeneous vector field of degree $k<0$, with domain of homogeneity $D \subset \mathbb{R}^{n}$, and that $0_{n}$ is a globally asymptotically stable equilibrium of $\dot{x}=$ $f(x)$. Then, the origin is a finite-time stable equilibrium of system (10) if

$$
\lim _{\varepsilon \rightarrow 0^{+}} \frac{\hat{f}_{i}\left(\delta_{\varepsilon}^{r}(x)\right)}{\varepsilon^{k+r_{i}}}=0
$$

$\forall i \in\{1, \ldots, n\}, \forall x \in S_{c}^{n-1}$

Proof: By Theorem 2.1, there exists a (globally) positive definite function $V \in \mathcal{C}^{1}\left(\mathbb{R}^{n}, \mathbb{R}\right)$ being $r$-homogeneous of degree $m>$ $\max \left\{r_{1}, \ldots, r_{n}\right\}$ such that $\frac{\partial V}{\partial x} f(x)$ is negative definite on $D$ and locally $r$-homogeneous of degree $m+k$ with domain of homogeneity $D$. From this and Lemma 2.1, we have that $\frac{\partial V}{\partial x} f(x) \leq-a V^{\alpha}(x)$, $\forall x \in\left\{\delta_{\varepsilon}^{r}(y) \in D: y \in S_{c}^{n-1}, \varepsilon \in(0,1)\right\}$, for some $a>0$ and $0<\alpha=(m+k) / m<1$. Let $\lambda=\inf _{x \in S_{c}^{n-1}} V^{\alpha}(x)>0$. Since $\frac{\partial V}{\partial x} \hat{f}\left(\delta_{\varepsilon}^{r}(x)\right)=O\left(\varepsilon^{m+k}\right)$ uniformly on $S_{c}^{n-1}$ as $\varepsilon \rightarrow 0$, there exists $\varepsilon_{0} \in(0,1)$ such that, for all $x \in S_{c}^{n-1}$ and every $\varepsilon \in\left(0, \varepsilon_{0}\right)$, we have that $\left|\frac{\partial V}{\partial x} \hat{f}\left(\delta_{\varepsilon}^{r}(x)\right)\right| \leq \frac{a \lambda}{2} \varepsilon^{m+k} \leq \frac{a}{2} \varepsilon^{m+k} V^{\alpha}(x)=$ $\frac{a}{2} V^{\alpha}\left(\delta_{\varepsilon}^{r}(x)\right)$, or equivalently $\left|\frac{\partial V}{\partial x} \hat{f}(x)\right| \leq \frac{a}{2} V^{\alpha}(x), \forall x \in \hat{D} \triangleq$ $\left\{\delta_{\varepsilon}^{r}(y) \in D: y \in S_{c}^{n-1}, \varepsilon \in\left(0, \varepsilon_{0}\right)\right\}$. Thus, $\frac{\partial V}{\partial x}(f+\hat{f})(x) \leq$ $-\frac{a}{2} V^{\alpha}(x), \forall x \in \hat{D}$, whence, by [9, Theorem 4.2] (see Appendix A), the origin is concluded to be a finite-time stable equilibrium of system (10).

\section{ApPLICATION TO THE GLOBAL FINITE-TIME STABILIZATION OF MANIPULATORS WITH BOUNDED INPUTS}

Global finite-time stabilization of robot manipulators with unbounded inputs was treated in [2] in the coordinate-dependent traditional framework of homogeneity. Here, we show how the PD-type result in [2] may be extended to the bounded input case through the local homogeneity analytical setting developed in the previous sections.

Let us begin by recalling the $n$-degree-of-freedom serial rigid robot manipulator dynamics [13]:

$$
H(q) \ddot{q}+C(q, \dot{q}) \dot{q}+g(q)=\tau
$$

where $q, \dot{q}, \ddot{q} \in \mathbb{R}^{n}$ are, respectively, the position (generalized coordinates), velocity, and acceleration vectors, $H(q) \in \mathbb{R}^{n \times n}$ is the inertia matrix, and $C(q, \dot{q}) \dot{q}, g(q), \tau \in \mathbb{R}^{n}$ are, respectively, the vectors of Coriolis and centrifugal, gravity, and external input generalized forces. Some well-known properties characterizing the terms of such a dynamical model are recalled here [13, Chap. 4]:

P1. $H(q)$ is a continuously differentiable matrix function:

a) being positive definite and bounded on $\mathbb{R}^{n}$, i.e. $\mu_{m} I_{n} \leq$ $H(q) \leq \mu_{M} I_{n}, \forall q \in \mathbb{R}^{n}$, for some positive constants $\mu_{m} \leq \mu_{M}$

b) such that $\|[H(x)-H(y)] z\| \leq k_{H}\|x-y\|\|z\|, \forall x, y, z \in$ $\mathbb{R}^{n}$, for some positive constant $k_{H}$.

$\mathrm{P} 2$. As a consequence of Property $\mathrm{P} 1$, the inverse matrix of $H(q)$, denoted $H^{-1}(q)$, exists and keeps the same analytical properties, i.e. $H^{-1}(q)$ is a continuously differentiable matrix function such that:

a) $\frac{1}{\mu_{M}} I_{n} \leq H^{-1}(q) \leq \frac{1}{\mu_{m}} I_{n}, \forall q \in \mathbb{R}^{n}$, where $\mu_{m}$ and $\mu_{M}$ are the positive constants characterized through Property P1a; b) $\left\|\left[H^{-1}(x)-H^{-1}(y)\right] z\right\| \leq \bar{k}_{H}\|x-y\|\|z\|, \forall x, y, z \in$ $\mathbb{R}^{n}$, for some constant $k_{H}>0$.

P3. With $\dot{H} \triangleq \frac{d}{d t} H, \dot{q}^{T}\left[\frac{1}{2} \dot{H}(q, \dot{q})-C(q, \dot{q})\right] \dot{q}=0, \forall(q, \dot{q}) \in$ $\mathbb{R}^{n} \times \mathbb{R}^{n}$.

P4. $g(q)$ is bounded on $\mathbb{R}^{n}$, or equivalently, every element of the gravity vector, $g_{i}(q)$, satisfies $\left|g_{i}(q)\right| \leq B_{g i}, \forall q \in \mathbb{R}^{n}$, for some positive constants $B_{g i}, i=1, \ldots, n$.

Property $\mathrm{P} 4$ is not satisfied by all types of robot manipulators but it is for instance by those having only revolute joints [13, §4.3]. Similarly, Property P1b, and consequently Property P2b, are known to be satisfied in the referred robotic context, i.e. by robots having only revolute joints [13, §4.1]. This section is addressed to manipulators satisfying Properties P4 and P2b.

Suppose that the absolute value of each input $\tau_{i}\left(i^{\text {th }}\right.$ element of the input vector $\tau$ ) is constrained to be smaller than a given saturation bound $T_{i}>0$, i.e. $\left|\tau_{i}\right| \leq T_{i}, i=1, \ldots, n$. In other words, letting $u_{i}$ represent the control signal (controller output) relative to the $i^{\text {th }}$ degree of freedom, we have that

$$
\tau_{i}=T_{i} \operatorname{sat}\left(\frac{u_{i}}{T_{i}}\right)
$$

$i=1, \ldots, n$, where $\operatorname{sat}(\cdot)$ is the standard saturation function, i.e. $\operatorname{sat}(\varsigma)=\operatorname{sign}(\varsigma) \min \{|\varsigma|, 1\}$. Let us note from (12)-(13) that $T_{i} \geq$ $B_{g i}$ (see Property $\left.\mathrm{P} 4\right), \forall i \in\{1, \ldots, n\}$, is a necessary condition for the manipulator to be stabilizable at any desired equilibrium configuration $q_{d} \in \mathbb{R}^{n}$. Thus, the following assumption turns out to be crucial within the analytical setting considered here:

Assumption 4.1: $T_{i}>B_{g i}, \forall i \in\{1, \ldots, n\}$.

The approach presented in this section involves special (saturation) functions fitting the following definition.

Definition 4.1: Given a positive constant $M$, a continuous function $\sigma: \mathbb{R} \rightarrow \mathbb{R}$ is said to be a generalized saturation with bound $M$ if it is a nondecreasing function being locally Lipschitz-continuous on $\mathbb{R} \backslash\{0\}$ and satisfying

(a) $\varsigma \sigma(\varsigma)>0$ for all $\varsigma \neq 0$;

(b) $|\sigma(\varsigma)| \leq M$ for all $\varsigma \in \mathbb{R}$.

Functions meeting Definition 4.1 have the next properties.

Lemma 4.1: Let $\sigma: \mathbb{R} \rightarrow \mathbb{R}$ be a generalized saturation function with bound $M$, and $k$ be a positive constant. Then

1. $\int_{0}^{\varsigma} \sigma(k r) d r>0, \forall \varsigma \neq 0$;

2. $\int_{0}^{\varsigma} \sigma(k r) d r \rightarrow \infty$ as $|\varsigma| \rightarrow \infty$.

Proof: See Appendix B.

Let us define the following control law

$$
u(q, \dot{q})=-s_{1}\left(K_{1} \bar{q}\right)-s_{2}\left(K_{2} \dot{q}\right)+g(q)
$$

where $\bar{q}=q-q_{d}$, for any constant (desired equilibrium position) vector $q_{d} \in \mathbb{R}^{n} ; K_{j} \in \mathbb{R}^{n \times n}, j=1,2$, are positive definite diagonal matrices, i.e. $K_{j}=\operatorname{diag}\left[k_{j 1}, \ldots, k_{j n}\right]$ with $k_{j i}>0$ for all $i=$ $1, \ldots, n, j=1,2 ; s_{j}: \mathbb{R}^{n} \rightarrow \mathbb{R}^{n}: z \mapsto\left(\sigma_{j 1}\left(z_{1}\right), \ldots, \sigma_{j n}\left(z_{n}\right)\right)^{T}$, $j=1,2$, with $\sigma_{j i}(\cdot)$ being generalized saturations, with bounds $M_{j i}$, such that $\sigma_{j i}\left(z_{i}\right)=\operatorname{sign}\left(z_{i}\right)\left|z_{i}\right|^{\beta_{j}}$ on $D_{j i}=\left\{z_{i} \in \mathbb{R}:\left|z_{i}\right| \leq L_{j i}\right\}$, for some $L_{j i}>0$ such that $L_{j i}^{\beta_{j}} \leq M_{j i}, i=1, \ldots, n, j=1,2$, and with $\beta_{j}$ and $M_{j i}$ satisfying

$$
\begin{gathered}
0<\beta_{1}<1 \quad, \quad \beta_{2}=\frac{2 \beta_{1}}{1+\beta_{1}} \\
M_{1 i}+M_{2 i}<T_{i}-B_{g i}
\end{gathered}
$$

$i=1, \ldots, n$ (observe that Assumption 4.1 ensures positivity of the right-hand side of inequalities (15b)).

Proposition 4.1: Consider system (12)-(13) taking $u=u(q, \dot{q})$ as defined in Eq. (14), under the satisfaction of Assumption 4.1 and 
the conditions stated through expressions (15). Thus, for any positive definite diagonal matrices $K_{j}, j=1,2$, global finite-time stability of the closed loop trivial solution $\bar{q}(t) \equiv 0_{n}$ is guaranteed with $\left|\tau_{i}(t)\right|=\left|u_{i}(t)\right|<T_{i}, i=1, \ldots, n, \forall t \geq 0$.

Proof: Observe that under the satisfaction of inequalities (15b) and the consideration of Property $\mathrm{P} 4$, we have that $\left|u_{i}(q, \dot{q})\right|=\mid-$ $\sigma_{1 i}\left(k_{1 i} \bar{q}_{i}\right)-\sigma_{2 i}\left(k_{2 i} \dot{q}_{i}\right)+g_{i}(q) \mid \leq M_{1 i}+M_{2 i}+B_{g i}<T_{i}, i=$ $1, \ldots, n, \forall(q, \dot{q}) \in \mathbb{R}^{n} \times \mathbb{R}^{n}$. From this and (13) one sees that, along the closed-loop system trajectories, $T_{i}>\left|u_{i}(t)\right|=\left|\tau_{i}(t)\right|$, $i=1, \ldots, n, \forall t \geq 0$. This proves that, under the scheme defined through Eq. (14), input saturation is avoided. Thus, the closed-loop system takes the form

$$
H\left(\bar{q}+q_{d}\right) \ddot{q}+C\left(\bar{q}+q_{d}, \dot{q}\right) \dot{q}=-s_{1}\left(K_{1} \bar{q}\right)-s_{2}\left(K_{2} \dot{q}\right)
$$

By defining $x_{1}=\bar{q}$ and $x_{2}=\dot{q}$, the closed-loop dynamics adopts the $2 n$-th order state-space representation

$$
\begin{aligned}
& \dot{x}_{1}=x_{2} \\
& \dot{x}_{2}=H^{-1}\left(x_{1}+q_{d}\right)\left[-C\left(x_{1}+q_{d}, x_{2}\right) x_{2}-s_{1}\left(K_{1} x_{1}\right)\right. \\
& \left.-s_{2}\left(K_{2} x_{2}\right)\right]
\end{aligned}
$$

By further defining $x=\left(x_{1}^{T}, x_{2}^{T}\right)^{T}$, these state equations may be rewritten in the form of system (10) with

$$
f(x)=\left(\begin{array}{c}
x_{2} \\
H^{-1}\left(q_{d}\right)\left[-s_{1}\left(K_{1} x_{1}\right)-s_{2}\left(K_{2} x_{2}\right)\right]
\end{array}\right)
$$

and

$$
\hat{f}(x)=\left(\begin{array}{c}
0_{n} \\
-H^{-1}\left(x_{1}+q_{d}\right) C\left(x_{1}+q_{d}, x_{2}\right) x_{2} \\
+\mathcal{H}\left(x_{1}\right)\left[-s_{1}\left(K_{1} x_{1}\right)-s_{2}\left(K_{2} x_{2}\right)\right]
\end{array}\right)
$$

where $\mathcal{H}\left(x_{1}\right)=H^{-1}\left(x_{1}+q_{d}\right)-H^{-1}\left(q_{d}\right)$. For every $\ell \in\{0,1\}$, let us define the continuously differentiable scalar function

$$
V_{\ell}(x)=\frac{1}{2} x_{2}^{T} H\left(\ell x_{1}+q_{d}\right) x_{2}+\int_{0_{n}}^{x_{1}} s_{1}^{T}\left(K_{1} r\right) d r
$$

where $\int_{0_{n}}^{x_{1}} s_{1}^{T}(r) d r=\sum_{i=1}^{n} \int_{0}^{x_{1 i}} \sigma_{1 i}\left(r_{i}\right) d r_{i}$. Observe that by Property P1a, we have that

$$
V_{\ell}(x) \geq \frac{\mu_{m}}{2}\left\|x_{2}\right\|_{2}^{2}+\int_{0_{n}}^{x_{1}} s_{1}^{T}\left(K_{1} r\right) d r
$$

$\ell=0,1$, whence, in view of Lemma 4.1, one sees that $V_{\ell}(x), \ell=$ 0,1 , are radially unbounded positive definite functions. Note further that, for every $\ell \in\{0,1\}$, we have

$$
\begin{aligned}
\dot{V}_{\ell}(x)= & \frac{\partial V_{\ell}}{\partial x}[f+\ell \hat{f}](x) \\
= & x_{2}^{T}\left[-s_{1}\left(K_{1} x_{1}\right)-s_{2}\left(K_{2} x_{2}\right)-\ell C\left(x_{1}+q_{d}, x_{2}\right) x_{2}\right] \\
& +\frac{\ell}{2} x_{2}^{T} \dot{H}\left(x_{1}+q_{d}, x_{2}\right) x_{2}+s_{1}^{T}\left(K_{1} x_{1}\right) x_{2} \\
= & -x_{2}^{T} s_{2}\left(K_{2} x_{2}\right)=-\sum_{i=1}^{n} x_{2 i} \sigma_{2 i}\left(k_{2 i} x_{2 i}\right)
\end{aligned}
$$

where, in the case of $\ell=1$, Property P3 has been applied. From this and point (a) of Definition 3.1, one sees that $Z_{\ell} \triangleq\{x \in$ $\left.\mathbb{R}^{2 n}: \dot{V}_{\ell}(x)=0\right\}=\left\{\left(x_{1}, x_{2}\right) \in \mathbb{R}^{n} \times \mathbb{R}^{n}: x_{2}=0_{n}\right\}$, $\ell=0,1$. Furthermore, for every $\ell \in\{0,1\}$, by analyzing the system dynamics $\dot{x}=f(x)+\ell \hat{f}(x)$ on $Z_{\ell}$ (whence it is seen that any solution with initial condition on $\left\{\left(x_{1}, x_{2}\right) \in Z_{\ell}: x_{1} \neq 0_{n}\right\}$ leaves $Z_{\ell}$ ), one sees that the only invariant subset of $Z_{\ell}$ is $\left\{0_{2 n}\right\}$. Hence, by the invariance theory $[14, \S 7.2], 0_{2 n}$ is concluded to be a globally asymptotically stable equilibrium of both the state equation $\dot{x}=f(x)$ and the closed-loop system $\dot{x}=f(x)+\hat{f}(x)$. Now, let $r_{c}=\left(r_{11}, \ldots, r_{1 n}, r_{21}, \ldots, r_{2 n}\right)^{T}$ with $r_{1 i}=r_{1}=2$ and $r_{2 i}=r_{2}=1+\beta_{1}, \forall i \in\{1, \ldots, n\}$. Based on Definition 2.1 and expressions (15a), one easily verifies that $f(x)$ is locally $r_{c^{-}}$ homogenous of degree $k=\beta_{1}-1<0$ with domain of homogeneity $D_{c}=D_{11} \times \cdots \times D_{1 n} \times D_{21} \times \cdots \times D_{2 n}$. Hence, by Theorem 3.1, $0_{2 n}$ is concluded to be a globally finite-time stable equilibrium of the state equation $\dot{x}=f(x)$. Thus, by Lemmas 3.2 and 3.1, global finitetime stability of $0_{2 n}$, as an equilibrium of the closed-loop system $\dot{x}=f(x)+\hat{f}(x)$, is concluded if $\lim _{\varepsilon \rightarrow 0^{+}} \hat{f}_{i}\left(\delta_{\varepsilon}^{r_{c}}(x)\right) / \varepsilon^{k+r_{i}}=0$ for every $i=1, \ldots, 2 n$ and all $x \in S_{a}^{2 n-1}=\left\{x \in \mathbb{R}^{2 n}:\|x\|=a\right\}$, for some $a>0$ such that $S_{a}^{2 n-1} \subset D_{c}$. Observe, from (16) and the definition of $r_{c}$, that this is the case if

$$
\begin{gathered}
\lim _{\varepsilon \rightarrow 0^{+}} \frac{1}{\varepsilon^{k+r_{2}}} \| H^{-1}\left(\varepsilon^{r_{1}} x_{1}+q_{d}\right) C\left(\varepsilon^{r_{1}} x_{1}+q_{d}, \varepsilon^{r_{2}} x_{2}\right) \varepsilon^{r_{2}} x_{2} \\
+\mathcal{H}\left(\varepsilon^{r_{1}} x_{1}\right)\left[s_{1}\left(K_{1} \varepsilon^{r_{1}} x_{1}\right)+s_{2}\left(K_{2} \varepsilon^{r_{2}} x_{2}\right)\right] \|=0
\end{gathered}
$$

Now, recalling that $r_{1}=2, r_{2}=1+\beta_{1}, k=\beta_{1}-1,0<\beta_{1}<1$, $\beta_{2}=2 \beta_{1} /\left(1+\beta_{1}\right)$, and $\sigma_{j i}(\varsigma)=\operatorname{sign}(\varsigma)|\varsigma|^{\beta_{j}}, \forall \varsigma \in D_{j i}, i=$ $1, \ldots, n, j=1,2$, we have that, for any $x \in S_{a}^{2 n-1} \subset D_{c}$,

$$
\begin{array}{r}
\lim _{\varepsilon \rightarrow 0^{+}} \frac{\left\|H^{-1}\left(\varepsilon^{2} x_{1}+q_{d}\right) C\left(\varepsilon^{2} x_{1}+q_{d}, \varepsilon^{1+\beta_{1}} x_{2}\right) \varepsilon^{1+\beta_{1}} x_{2}\right\|}{\varepsilon^{2 \beta_{1}}} \\
=\left\|H^{-1}\left(q_{d}\right) C\left(q_{d}, 0_{n}\right) x_{2}\right\| \lim _{\varepsilon \rightarrow 0^{+}} \varepsilon^{1-\beta_{1}}=0
\end{array}
$$

and, by Property P2b (recall that $\mathcal{H}\left(x_{1}\right)=H^{-1}\left(x_{1}+q_{d}\right)-$ $\left.H^{-1}\left(q_{d}\right)\right)$,

$$
\begin{aligned}
& \lim _{\varepsilon \rightarrow 0^{+}} \frac{\left\|\mathcal{H}\left(\varepsilon^{2} x_{1}\right)\left[s_{1}\left(K_{1} \varepsilon^{2} x_{1}\right)+s_{2}\left(K_{2} \varepsilon^{1+\beta_{1}} x_{2}\right)\right]\right\|}{\varepsilon^{2 \beta_{1}}} \\
& \leq \bar{k}_{H}\left\|x_{1}\right\|\left\|s_{1}\left(K_{1} x_{1}\right)+s_{2}\left(K_{2} x_{2}\right)\right\| \lim _{\varepsilon \rightarrow 0^{+}} \varepsilon^{2}=0
\end{aligned}
$$

Therefore, (17) is concluded to be satisfied, which completes the proof.

The proposed globally finite-time stabilizing scheme may be implemented by defining, for instance, $\sigma_{j i}(\varsigma)=\operatorname{sign}(\varsigma) \min \left\{|\varsigma|^{\beta_{j}}, M_{j i}\right\}$, $i=1, \ldots, n, j=1,2$.

\section{Conclusions}

A local notion of homogeneity has been formally defined. Relevant results originally stated in the framework of homogeneous functions and vector fields have been reformulated in the proposed local context. In particular, global finite-time stability has been characterized in the developed local framework. The proposed analytical setting was applied to develop a generalized scheme for the global finitetime stabilization of robot manipulators with bounded inputs. More generally, the proposed analytical framework and results state an alternative platform for the analysis of global finite-time stability and the synthesis of global finite-time stabilizers that relaxes constraints imposed or generated by homogeneity requirements.

\section{APPENDIX}

\section{A. Some cited results}

For the sake of ease on the reading, some results cited from other works are reproduced here. The first four statements concern theorems or lemmas applied in the proofs of the results developed in this study. The last four are relevant results from the traditional homogeneity and/or finite-time stability frameworks whose reformulation in the proposed local context is presented in this work. 
[7, Theorem 1]: If a continuous vector field $f: \mathbb{R}^{n} \rightarrow \mathbb{R}^{n}$ is such that $f\left(0_{n}\right)=0_{n}$, the trivial solution of the equation $\dot{x}=f(x)$ is strongly stable in $\mathbb{R}^{n}$ if and only if there exists a (so-called Lyapunov) function $V \in \mathcal{C}^{\infty}\left(\mathbb{R}^{n}, \mathbb{R}\right)$ such that $V\left(0_{n}\right)=0$, $V(x)>0$ for all $x \neq 0_{n}, V(x) \rightarrow+\infty$ if $\|x\| \rightarrow+\infty$ and $\frac{\partial V}{\partial x} f(x)<0, \forall x \neq 0_{n}$.

[1, Lemma 2.2]: Let $\psi: \mathbb{R}_{>0} \times \mathbb{R}^{n} \rightarrow \mathbb{R}^{n}$ be the continuous global semiflow of a system $\Sigma: \dot{x}=f(x)$ [with $f$ being a continuous vector field on $\mathbb{R}^{n}$ with the property that, for every initial condition $x(0)=x_{0} \in \mathbb{R}^{n}$, the system $\Sigma$ has a unique right-maximally-defined solution $x(t)$ defined on $[0, \infty)]$. Suppose $\mathcal{K} \subset \mathbb{R}^{n}$ is nonempty, compact and attractive under $\psi$, and let $\mathcal{M}$ be a nonempty and compact subset of the domain of attraction of $\mathcal{K}$. Then $\psi\left(\mathbb{R}_{\geq 0} \times \mathcal{M}\right)$ is bounded. In addition, if $\mathcal{K}$ is asymptotically stable under $\psi$, then, for every open neighborhood $\mathcal{U}$ of $\mathcal{K}$, there exists $\tau>0$ such that $\psi(t, \mathcal{M}) \subset \mathcal{U}$ for all $t>\tau$.

Next statement is a coordinate-dependent partial version of:

[1, Theorem 4.1]: Suppose $V: \mathbb{R}^{n} \rightarrow \mathbb{R}$ is continuous on $\mathbb{R}^{n} \backslash$ $\left\{0_{n}\right\}$ and $r$-homogeneous of degree $l$ :

- if $l<0$, then $V$ is continuous on $\mathbb{R}^{n}$ if and only if $V \equiv 0$;

- if $l=0$, then $V$ is continuous on $\mathbb{R}^{n}$ if and only if $V \equiv V\left(0_{n}\right)$;

- if $l>0$, then $V$ is continuous on $\mathbb{R}^{n}$.

[9, Theorem 4.2]: Consider the system $\Sigma: \dot{x}=f(x)$, where $f: \mathcal{D} \rightarrow \mathbb{R}^{n}$ is continuous on an open neighborhood $\mathcal{D} \subseteq \mathbb{R}^{n}$ of the origin and $f\left(0_{n}\right)=0_{n}$. Suppose there exists a continuous positive definite function $V: \mathcal{D} \rightarrow \mathbb{R}$ such that $\dot{V}(x)+c[V(x)]^{\alpha} \leq 0$, $\forall x \in \mathcal{V} \backslash\left\{0_{n}\right\}$, for some real numbers $c>0$ and $\alpha \in(0,1)$ and an open neighborhood $\mathcal{V} \subset \mathcal{D}$ of the origin. Then the origin is a finite-time stable equilibrium of system $\Sigma$. Moreover, with $\mathcal{N}$ as in Definition 3.1, the settling-time function $T$ satisfies $T(x) \leq\left[(V(x))^{1-\alpha}\right] /[c(1-\alpha)], \forall x \in \mathcal{N}$. If in addition $\mathcal{D}=\mathbb{R}^{n}$, $V$ is proper, and $\dot{V}$ takes negative values on $\mathbb{R}^{n} \backslash\left\{0_{n}\right\}$, then the origin is a globally finite-time stable equilibrium of system $\Sigma$.

[3, Theorem 5.8]: Let $f$ be a continuous vector field on $\mathbb{R}^{n}$ such that the origin is a locally asymptotically stable equilibrium of the $n$-th order autonomous system $\Sigma: \dot{x}=f(x)$. Assume that $f$ is $r$-homogeneous of degree $\alpha$ for some $r \in \mathbb{R}_{>0}^{n}$. Then, for any positive integer $p$ and any $m>p \cdot \max \left\{r_{1}, \ldots, r_{n}\right\}$, there exists a strict Lyapunov function $V$ for $\Sigma$ which is $r$-homogeneous of degree $\alpha$ and $V \in \mathcal{C}^{p}\left(\mathbb{R}^{n}, \mathbb{R}\right)$. As a direct consequence the time-derivative $\dot{V}(x)=\frac{\partial V}{\partial x} f(x)$ is $r$-homogeneous of degree $m+\alpha$.

The next statement is a coordinate-dependent version of:

[1, Theorem 7.1]: Suppose $f: \mathbb{R}^{n} \rightarrow \mathbb{R}^{n}$ is $r$-homogeneous of degree $m$ for some $r \in \mathbb{R}_{>0}^{n}$. Then, the origin is a finite-time stable equilibrium of system $\Sigma: \dot{x}=f(x)$ if and only if it is an asymptotically stable equilibrium of $\Sigma$ and $m<0$.

The next statement is a coordinate-dependent version of:

[1, Lemma 4.2]: Suppose $V_{1}$ and $V_{2}$ are continuous real-valued functions on $\mathbb{R}^{n}, r$-homogeneous of degrees $\alpha_{1}>0$ and $\alpha_{2}>0$, respectively, for some $r \in \mathbb{R}_{>0}^{n}$, and $V_{1}$ is positive definite. Then, for every $x \in \mathbb{R}^{n}, c_{1}\left[V_{1}(x)\right]^{\alpha_{2} / \alpha_{1}} \leq V_{2}(x) \leq c_{2}\left[V_{1}(x)\right]^{\alpha_{2} / \alpha_{1}}$, with $c_{1}=\min _{\left\{z: V_{1}(z)=1\right\}} V_{2}(z)$ and $c_{2}=\max _{\left\{z: V_{1}(z)=1\right\}} V_{2}(z)$.

[12, Lemma 3]: Consider the system $\Sigma: \dot{x}=f(x)+\hat{f}(x), x \in \mathbb{R}^{n}$, where $f(x)$ is $n$-dimensional continuous $r$-homogeneous vector field of degree $k<0$, for some $r \in \mathbb{R}_{>0}^{n}$, satisfying $f\left(0_{n}\right)=0_{n}$, and $\hat{f}$ is also a continuous vector field satisfying $\hat{f}\left(0_{n}\right)=0_{n}$. Assume the zero solution of $\dot{x}=f(x)$ is asymptotically stable. Then, the zero solution of $\Sigma$ is locally finite-time stable if $\lim _{\varepsilon \rightarrow 0^{+}} \hat{f}_{i}\left(\delta_{\varepsilon}^{r}\right) / \varepsilon^{k+r_{i}}=$ $0, i=1, \ldots, n$, uniformly for any $x \in \mathbb{R}^{n}$ such that $\|x\|=1$.

\section{B. Proof of Lemma 4.1}

1. From the nondecreasing character of $\sigma$, and its satisfaction of point (a) of Definition 4.1, we have that there exist constants $a>0, k_{a}>0$, and $c \geq 1$ such that $|\sigma(\varsigma)| \geq k_{a}|a \operatorname{sat}(\varsigma / a)|^{c}$. Hence

$$
S_{a}(\varsigma) \triangleq \int_{0}^{\varsigma} \operatorname{sign}(r) k_{a}|a \operatorname{sat}(r / a)|^{c} d r \leq \int_{0}^{\varsigma} \sigma(k \varsigma) d r
$$

$\forall \varsigma \in \mathbb{R}$, with

$$
S_{a}(\varsigma)= \begin{cases}\frac{k_{a}}{c+1}|\varsigma|^{c+1} & \forall|\varsigma| \leq a \\ k_{a} a^{c}\left(|\varsigma|-\frac{a c}{c+1}\right) & \forall|\varsigma|>a\end{cases}
$$

whence it is clear that $\int_{0}^{\varsigma} \sigma(k \varsigma) d r>0, \forall \varsigma \neq 0$.

2. From expressions (18), one sees on the one hand that $\lim _{|\varsigma| \rightarrow \infty} S_{a}(\varsigma) \leq \lim _{|\varsigma| \rightarrow \infty} \int_{0}^{\varsigma} \sigma(k r) d r$, and on the other that $S_{a}(\varsigma) \rightarrow \infty$ as $|\varsigma| \rightarrow \infty$, whence we conclude that $\int_{0}^{\varsigma} \sigma(k r) d r \rightarrow \infty$ as $|\varsigma| \rightarrow \infty$.

\section{ACKNOWLEDGEMENT}

The authors thank the Associate Editor that handled the review process for his valuable comments that helped us to improve the presentation of our paper.

\section{REFERENCES}

[1] S.P. Bhat and D.S. Bernstein, "Geometric homogeneity with applications to finite-time stability," Mathematics of Control, Signals, and Systems, vol. 17, no. 2, pp. 101-127, 2005.

[2] Y. Hong, Y. Xu, and J. Huang: "Finite-time control for robot manipulators," Systems \& Control Letters, vol. 46, no. 4, pp. 243-253, 2002.

[3] A. Bacciotti and L. Rosier, Liapunov Functions and Stability in Control Theory, 2nd ed., Berlin: Springer, 2005.

[4] M.T. Frye, S. Ding, C. Qian, and S. Li, "Fast convergent observer design for output feedback stabilisation of a planar vertical takeoff and landing aircraft," IET Control Th. and App., vol. 4, no. 4, pp. 690-700, 2010.

[5] C. Quian, "A homogeneous domination approach for global output feedback stabilization of a class of nonlinear systems," Proc. American Control Conference, Portland, OR, 2005, pp. 4708-4715.

[6] D. Aeyels and P. de Leenheer, "Extension of the Perron-Frobenious theorem to homogeneous systems," SIAM Journal on Control and Optimization, vol. 41, no. 2, pp. 563-582, 2002.

[7] L. Rosier, "Homogeneous Lyapunov function for homogeneous continuous vector field," Syst. \& Cont. Lett., vol. 19, no. 6, pp. 467-473, 1992.

[8] H.K. Khalil, Nonlinear Systems, 3rd ed., Upper Saddle River, NJ: Prentice Hall, 2002.

[9] S.P. Bhat and D.S. Bernstein, "Finite-time stability of continuous autonomous systems," SIAM Journal on Control and Optimization, vol. 38, no. 3, pp. 751-766, 2000.

[10] N. Rouche, P. Habets, and M. Laloy, Stability Theory by Lyapunov's Direct Method, New York: Springer-Verlag, 1977.

[11] T. Yoshizawa, Stability theory by Liapunov's second method, Tokyo: The Mathematical Society of Japan, 1966.

[12] Y. Hong, J. Huang, and Y. Xu, "On an output feedback finite-time stabilization problem," IEEE Transactions on Automatic Control, vol. 46, no. 2, pp. 305-309, 2001

[13] R. Kelly, V. Santibáñez, and A. Loría, Control of Robot Manipulators in Joint Space, London: Springer, 2005.

[14] A.N. Michel, L. Hou, and D. Liu, Stability of Dynamical Systems, Boston: Birkhäuser, 2008. 